Revista Brasileira de Agricultura Irrigada v.11, nº.6, p. 1894 - 1903, 2017

ISSN 1982-7679 (On-line)

Fortaleza, CE, INOVAGRI - http://www.inovagri.org.br

DOI: $10.7127 /$ rbai.v11n600649

Protocolo 649.17 - 29/03/2017 Aprovado em 03/05/2017

\title{
EVAPOTRANSPIRAÇÃO DO GIRASSOL PELO MÉTODO DO TANQUE CLASSE “A” NA REGIÃO DO SEMIÁRIDO BRASILEIRO
}

\author{
Poliana de Caldas Pereira ${ }^{1}$, Alexandre Maniçoba da Rosa Ferraz Jardim², Lucivania \\ Rodrigues Lima ${ }^{3}$, Maria Gabriela de Queiroz ${ }^{4}$, Thieres George Freire da Silva ${ }^{5}$, José Edson \\ Florentino de Morais ${ }^{6}$
}

\section{RESUMO}

Objetivou-se com esta pesquisa estimar a evapotranspiração do girassol (Helianthus annuus L.), com diferentes valores do coeficiente do Tanque Classe "A” (Kp) na região do Vale do Pajeú, Pernambuco, Semiárido brasileiro. A pesquisa foi realizada em Serra Talhada, Pernambuco, empregando dados meteorológicos e do Tanque Classe "A" do período de outubro de 1999 a fevereiro de 2011. Com os valores médios mensais de Kp de sete métodos, obtidos dos anos ímpares do período de estudo estimou-se os valores de evapotranspiração de referência pelo método do Tanque Classe "A" (ETo(TCA)) e evapotranspiração da cultura do girassol pelo método do Tanque Classe "A” (ETc(TCA)), para os anos pares do mesmo período. Em sequência, os valores de $\mathrm{ETC}_{(\mathrm{TCA})}$ foram estimados para diferentes ciclos da cultura ao longo do ano e avaliados por meio do teste de Tukey, ao nível de $5 \%$ de probabilidade, e através de índices estatísticos, sempre considerando a evapotranspiração da cultura pelo método de PenmanMonteith FAO 56 (ЕTсрм), como referência. Os resultados mostraram que todos os valores de ETo(TCA), superestimaram a ETopm, tendo destaque ETo(TCA)Kpsnyder por apresentar a maior diferença. Para a simulações dos valores de $\mathrm{ETC}_{(\mathrm{TCA})}$ nos diferentes ciclos, os piores

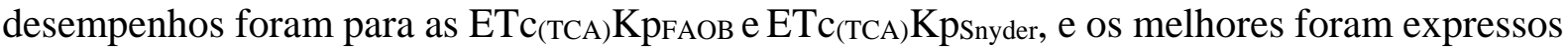

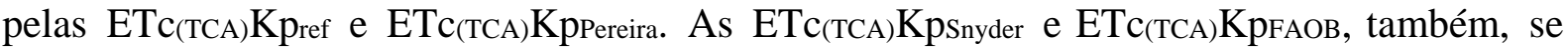
comportaram de forma insatisfatória quando foram submetidas aos índices estatísticos, superestimando as lâminas de irrigação. Já os resultados das ETc(TCA)Kpref e ETc(TCA)Kppereira tiveram os melhores desempenhos, assim recomenda-se o uso dos valores dos Kpref e Kppereira.

Palavras-chave: Helianthus annuus L., manejo de irrigação, métodos estatísticos

\footnotetext{
${ }^{1}$ Engenheira Agrônoma, Doutoranda em Meteorologia Agrícola, pela Universidade Federal de Viçosa (UFV), e-mail: po.caldas@hotmail.com 2 Técnico em Agropecuária, Engenheiro Agrônomo, Mestrando em Produção Vegetal, pela Unidade Acadêmica de Serra Talhada, Universidade Federal Rural de Pernambuco (UFRPE/UAST), e-mail: alexandremrfj@gmail.com

3 Bióloga, Mestre em Produção Vegetal, pela Unidade Acadêmica de Serra Talhada, Universidade Federal Rural de Pernambuco (UFRPE/UAST), e-mail: lucivania_rodrigues@hotmail.com

${ }^{4}$ Engenheira Agrônoma, Doutoranda em Meteorologia Agrícola, pela Universidade Federal de Viçosa (UFV), e-mail: mg.gabi@hotmail.com

${ }^{5}$ Engenheiro Agrônomo, Doutor em Meteorologia Agrícola, pela Universidade Federal de Viçosa (UFV), Professor Adjunto IV da Área de Meteorologia Aplicada à Ciências Agrárias, Ambientais e Exatas, e-mail: thieres_freire@yahoo.com.br

6 Engenheiro Agrônomo, Doutorando em Engenharia Agrícola, pela Universidade Federal Rural de Pernambuco (UFRPE), e-mail: joseedson50@hotmail.com
} 


\title{
EVAPOTRANSPIRATION OF THE SUNFLOWER BY THE CLASS “A” PAN METHOD IN THE BRAZILIAN SEMI-ARID REGION
}

\begin{abstract}
The objective of this study was to estimate the evapotranspiration of sunflower (Helianthus annuus L.), with different coefficient values of Class-A pan coefficient (Kp) in the Pajeú Valley, Pernambuco, Brazil semi-arid region. The survey was conducted in Serra Talhada, Pernambuco, using meteorological data and Class-A pan from October 1999 to February 2011. With the average monthly values of Kp seven methods, obtained from the odd years of the study period, it estimated the evapotranspiration reference by method Class-A pan (ETo(TCA)) and sunflower crop evapotranspiration by method Class-A pan ( $\left.\mathrm{ETC}_{(\mathrm{TCA})}\right)$ for the same period even years. In sequence, the values ETc(TCA) were estimated for different crop cycles throughout the year and assessed by the Tukey test, at 5\% probability, and using statistical indices, always considering the crop evapotranspiration by Penman-Monteith FAO 56 (ЕTсPM) as a reference. The results showed that all values ETo(TCA), overestimated ETopm, having featured

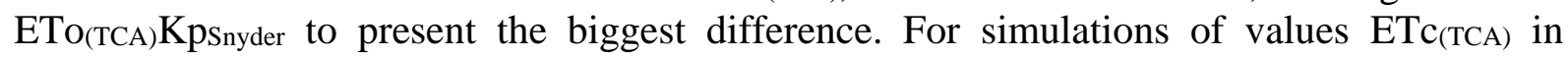
different cycles, the worst performers were for ETc(ТСA)Kрғаов and ETc(TCA)Kpsnyder, and the

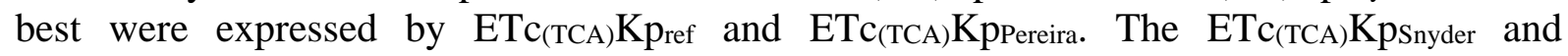
ETс(ТСA)Крғаов also behaved unsatisfactorily when they were subjected to statistical indices, overestimating the irrigation levels. Already the result of $\mathrm{ETC}_{(\mathrm{TCA})} \mathrm{Kp}_{\text {ref }}$ and $\mathrm{ETC}_{\text {(TCA)Kppereira }}$ had the best performance, so it is recommended to use the values of Kpref and Kppereira.
\end{abstract}

Keywords: Helianthus annuus L., irrigation management, statistical methods

\section{INTRODUÇÃO}

Com a escassez dos recursos hídricos no mundo, principalmente em regiões áridas e semiáridas, é indispensável a otimização do uso da água afim de atender às necessidades humanas, industriais e da agricultura. Tratando-se da irrigação, objetivando obter um manejo hídrico eficiente, é conveniente o conhecimento das necessidades hídricas das culturas, sendo considerando como componente chave para esse problema o conhecimento de suas evapotranspirações (ET) (ALCARAS et al., 2016).

A ET trata-se da combinação de dois processos separados, sendo que em um deles a água é perdida da superfície do solo por evaporação e no outro é perdida pelas plantas através da transpiração (ALLEN et al., 1998).

A evapotranspiração da cultura (ETc) é um elemento indispensável no dimensionamento do manejo hídrico de diferentes espécies, sendo que está representa a quantidade de água que deve ser reposta ao solo para manter o desenvolvimento e produtividade das plantas (PEREIRA et al., 2013). Desta forma, a otimização no manejo de irrigação está na quantificação da água a ser aplicada a uma determinada cultura, que é comumente associada à capacidade da superfície do solo e da vegetação de perder água para a atmosfera (ESTEVES et al., 2009; ALCARAS et al., 2016).

Para a obtenção da ETc é necessário a utilização do coeficiente da cultura (Kc), que varia com a espécie e o estádio de desenvolvimento; e a estimativa da evapotranspiração de referência (ETo), processo onde uma superfície padrão gramada, sem restrição de umidade, perde água para a atmosfera (PEREIRA et al., 2013).

Na literatura há diferentes métodos de obtenção da ETo que necessitam de muitas variáveis meteorológicas como o método de Penman-Monteith, contudo, existe métodos mais simples, que relaciona-se a um só componente, como é o caso do método do Tanque Classe “A” que utiliza medições de evaporação da água (SOUSA et al., 2010). 


\section{SEMIÁRIDO BRASILEIRO}

Uma das dificuldades na obtenção da ETo por métodos dependentes de muitos elementos meteorológicos é a ausência de dados, desta forma, metodologias que não necessitem de tantas informações atmosféricas é uma alternativa viável, enquadrando entre essas alternativas o método do Tanque Classe “A”, que utiliza fatores climáticos (radiação solar, vento, temperatura e umidade relativa do ar) sobre a evaporação da lâmina de água livre (OLIVEIRA et al., 2008).

Além dos dados de evaporação, este método é dependente do coeficiente do Tanque Classe “A” (Kp), o qual é determinado por metodologias fundamentadas em valores médios de umidade relativa do ar, velocidade do vento, tamanho da bordadura e no tipo de superfície em que o tanque se encontra (PEREIRA et al., 2014). Também pode-se assumir as resistências da superfície e aerodinâmica (CUENCA, 1989; SNYDER, 1992; PEREIRA et al., 1995; ALLEN et al., 1998), para a sua determinação, ou simplesmente adotar de um único valor de Kp para todo o período do ano (DOORENBOS; PRUITT, 1977).

A determinação da ETc vem sendo cada vez mais aplicada na agricultura. E na região semiárida do Nordeste brasileiro, onde quase todos os períodos do ano necessitam da irrigação para a obtenção de produtos agrícolas, essa metodologia auxilia no uso eficiente da água na produção, tornando-se possível o cultivo de diferentes espécies e diminuindo o desperdício hídrico (CAVALCANTE JUNIOR et al., 2013; ALCARAS et al., 2016).

Devido a aplicação da técnica de irrigação, culturas como o girassol (Helianthus annuus L.) podem ser cultivadas durante todo o ano na região semiárida do Nordeste, uma vez que o seu desenvolvimento é afetado em situações de deficiência hídrica dos solos (SILVA et al., 2007; FREITAS et al., 2012). Neste contexto, o presente trabalho teve como objetivo estimar a evapotranspiração da cultura do girassol (Helianthus annuus L.), utilizando diferentes valores de Kp para a região do Vale do Pajeú, Pernambuco.

\section{MATERIAIS E MÉTODOS}

A área estudada abrange a região do Vale Pajeú, localizada no município de Serra Talhada, Pernambuco (Lat. $7^{\circ} 59^{\prime}$ S; Long. $38^{\circ} 15^{\prime}$ O e Alt. $431 \mathrm{~m}$ ), que apresenta um clima do tipo BSwh' (com incidência de chuva na maioria nos meses mais quentes, e seca nos meses frios do ano), conforme a classificação de Köppen (ALVARES et al., 2013) (Figura 1).

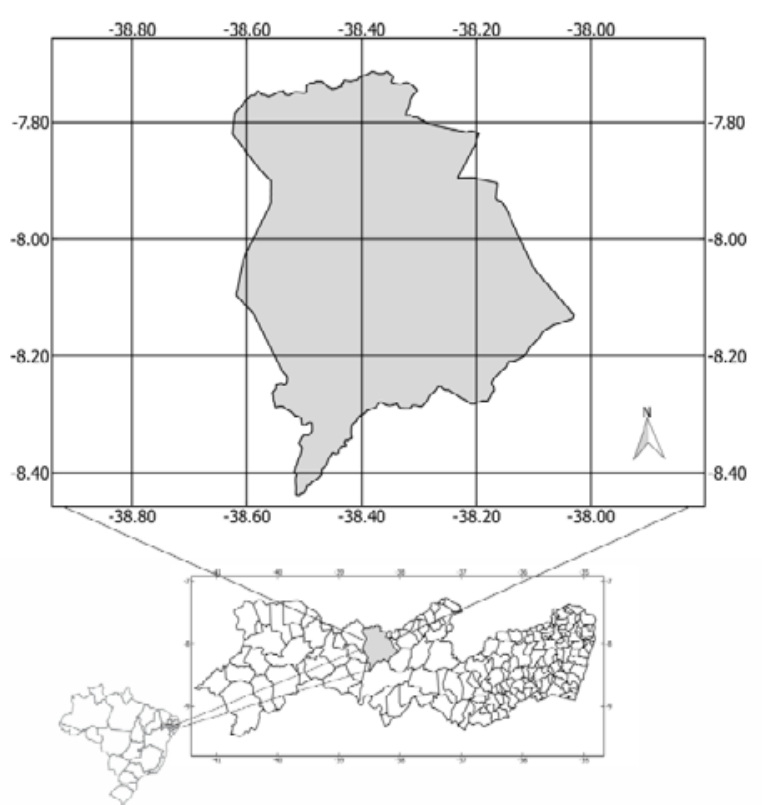

Figura 1. Localização geográfica da área de estudo. 
Esta pesquisa compreendeu o período de outubro de 1999 a fevereiro de 2011, o qual possui grande similaridade em relação aos valores da normal climatológica (1961-1990), indicando que a demanda atmosférica e a reposição de água durante o período de análise são representativas das condições climatológicas da região, permitindo a definição de valores médios de Kp ajustados localmente, conforme definidos por
Pereira et al. (2014).

A partir dos valores médios mensais do coeficiente do Tanque Classe “A” (Kp), determinados por Pereira et al. (2014), para Serra Talhada por sete métodos - Kpref, Pereira et al. (2014); Snyder (1992); Cuenca (1989); Pereira et al. (1995); FAO caso A e FAO caso B (ALLEN et al., 1998); e Doorenbos e Pruitt (1977) nos anos ímpares do período de estudo (Tabela 1).

Tabela 1. Média mensal dos valores do coeficiente do tanque Classe “A” (Kp), calculados por meio de sete métodos: Kpref, Pereira et al. (2014); Kpsnyder, Snyder (1992); Kpcuenca, Cuenca (1989); Kppereira, Pereira et al. (1995); Kрғаод e Kрғаов, Allen et al. (1998); e Kpd\&p, Doorenbos e Pruitt (1977), para a série de dados dos anos impares, entre 1999 e 2011, em Serra Talhada, Pernambuco.

\begin{tabular}{cccccccc}
\hline Meses & $\mathbf{K p}_{\text {ref }}$ & $\mathbf{K p}_{\text {snyder }}$ & $\mathbf{K}_{\text {Cuenca }}$ & $\mathbf{K p}_{\text {Pereira }}$ & $\mathbf{K}_{\text {FAOA }}$ & $\mathbf{K}_{\text {FAOB }}$ & $\mathbf{K}_{\text {D\&P }}$ \\
\hline Jan. & 0,732 & 0,898 & 0,726 & 0,725 & 0,700 & 0,817 & 0,750 \\
Fev. & 0,696 & 0,844 & 0,770 & 0,715 & 0,735 & 0,867 & 0,750 \\
Mar. & 0,805 & 0,830 & 0,773 & 0,720 & 0,750 & 0,877 & 0,750 \\
Abr. & 0,752 & 0,855 & 0,755 & 0,720 & 0,747 & 0,880 & 0,750 \\
Mai. & 0,688 & 0,846 & 0,686 & 0,700 & 0,750 & 0,890 & 0,750 \\
Jun. & 0,640 & 0,832 & 0,775 & 0,690 & 0,727 & 0,873 & 0,750 \\
Jul. & 0,667 & 0,850 & 0,770 & 0,685 & 0,723 & 0,867 & 0,750 \\
Ago. & 0,656 & 0,872 & 0,744 & 0,680 & 0,707 & 0,850 & 0,750 \\
Set. & 0,690 & 0,918 & 0,716 & 0,690 & 0,687 & 0,817 & 0,750 \\
Out. & 0,640 & 0,936 & 0,702 & 0,702 & 0,680 & 0,796 & 0,750 \\
Nov. & 0,660 & 0,935 & 0,703 & 0,720 & 0,690 & 0,800 & 0,750 \\
Dez. & 0,647 & 0,923 & 0,703 & 0,716 & 0,693 & 0,806 & 0,750 \\
\hline Anual & 0,688 & 0,879 & 0,737 & 0,696 & 0,715 & 0,846 & 0,750 \\
\hline
\end{tabular}

Fonte: Pereira et al. (2014).

Foi determinado a evapotranspiração de referência pelo método do Tanque Classe "A" (ETo(TCA)) e a evapotranspiração da cultura do girassol (ETc), para os anos pares do mesmo período, conforme Equações 1 e 2, respectivamente:

$$
\begin{gathered}
E T o_{(T C A)}=E T C A \cdot K p_{(x)} \\
E T C_{(T C A)}=E T o_{(T C A)} \cdot K C
\end{gathered}
$$

em que,

$\mathrm{ETo}_{(\mathrm{TCA})}=$ evapotranspiração de referência obtida usando os dados de Kp propostos pelos métodos ( $\left.\mathrm{mm} \mathrm{dia}^{-1}\right)$;
ETCA = evaporação do Tanque Classe "A", adquirida na Universidade Federal Rural de Pernambuco - Unidade Acadêmica de Serra Talhada (UFRPE/UAST), para o período de estudo;

$\mathrm{Kp}(\mathrm{x})=$ coeficiente do Tanque Classe "A", referente aos valores de Kp dos sete métodos (decimal);

Kc = coeficiente de cultura, para cada uma das fases fenológicas do girassol (CAVALCANTE JUNIOR et al., 2013).

Sendo considerado como Kp de referência os valores de Kpref, devido Pereira et al. (2014), terem ajustado esse coeficiente pela relação entre a evapotranspiração de referência pelo método de Penman-Monteith (ETopm) com a evaporação do Tanque Classe "A" 
(ETCA), dando maior confiabilidade a esses valores, por serem obtidos de uma grande quantidade de dados meteorológicos locais.

Os valores de ETc da cultura do girassol, estimados pelos dados médios mensais dos Kp (Tabela 1), foram simulados para quatro ciclos possíveis de 90 dias ao longo do ano, afim de observar as diferentes demandas hídricas durante o ano, sendo esses valores avaliados pelo teste de Tukey ao nível de $5 \%$ de probabilidade.

Também foi analisado o desempenho dos valores de ETc conforme Equações 3, 4, 5, 6 e 7, por índices estatísticos de precisão (coeficiente de correlação, r); exatidão (índice de concordância, d); estimativas dos erros estatísticos (erro médio de estimativa - MBE e a raiz quadrada do quadrado do erro médio RQME); e índice de confiança (c), resultante do produto entre o coeficiente de correlação (r) e o índice de concordância (d), sendo último conforme Camargo e Sentelhas (1997):

$\mathrm{r}=\frac{\left[\sum \mathrm{ETc}_{(\mathrm{TCA})}\left(\mathrm{ETc}_{\mathrm{PM}}-\overline{\mathrm{ETc}_{\mathrm{PM}}}\right)\right]}{\left[\sum_{\mathrm{i}=1}^{\mathrm{n}}\left(\mathrm{ETc}_{\mathrm{PM}}-\overline{\mathrm{ETc}_{\mathrm{PM}}}\right)^{2} \sum_{\mathrm{i}=1}^{\mathrm{n}}\left(\mathrm{ETc}_{(\mathrm{TCA})}-\overline{\mathrm{ETc}_{(\mathrm{TCA})}}\right)^{2}\right]^{112}}$

$\mathrm{d}=1-\left[\frac{\sum_{\mathrm{i}=1}^{\mathrm{n}}\left(\mathrm{ETc}_{(\mathrm{TCA})}-\mathrm{ETc}_{\mathrm{PM}}\right)^{2}}{\sum_{\mathrm{i}=1}^{\mathrm{n}}\left(\left|\mathrm{ETC}_{(\mathrm{TCA})}-\overline{\mathrm{ETC}_{\mathrm{PM}}}\right|+\left|\mathrm{ETc}_{\mathrm{PM}}-\overline{\mathrm{ETc}_{\mathrm{PM}}}\right|\right)^{2}}\right]$

$$
\begin{gathered}
\mathrm{MBE}=\frac{1}{\mathrm{n}} \sum_{\mathrm{i}=1}^{\mathrm{n}}\left(\mathrm{ETC}_{(\mathrm{TCA})}-\mathrm{ETC}_{\mathrm{PM}}\right) \\
\mathrm{RMSE}=\left[\frac{1}{\mathrm{n}} \sum_{\mathrm{i}=1}^{\mathrm{n}}\left(\mathrm{ETC}_{(\mathrm{TCA})}-\mathrm{ETC}_{\mathrm{PM}}\right)^{2}\right]^{112} \\
\mathrm{c}=\mathrm{d} \cdot \mathrm{r}
\end{gathered}
$$

em que,

$\mathrm{n}$ = número de observações;

ETcpm $=$ valores acumulados da evapotranspiração da cultura (mm dia $\left.{ }^{-1}\right)$, obtidos com a ETopm (evapotranspiração de referência pelo método de Penman-Monteith) e o Kc.
Sendo ETcpm considerada como o método de referência de evaporação da cultura, esta pode ser determinada pela Equação 8:

$$
E T c_{P M}=E T o_{P M} \cdot K C
$$

A estimativa da evapotranspiração de referência pelo método de Penman-Monteith FAO 56 (ETopm) foi calculada pela Equação 9, proposta por Allen et al. (1998):

$$
\mathrm{ETo}_{\mathrm{PM}}=\frac{0,408 \Delta(\mathrm{Rn}-\mathrm{G})+\gamma\left(\frac{900}{\mathrm{t}_{\text {méd }}+273}\right) \mathrm{u}_{2}\left(\mathrm{e}_{\mathrm{s}}-\mathrm{e}_{\mathrm{a}}\right)}{\Delta+\gamma\left(1+0,34 \mathrm{u}_{2}\right)}
$$

em que,

$\mathrm{R}_{\mathrm{n}}=$ radiação líquida à superfície $\left(\mathrm{MJ} \mathrm{m}^{-2} \mathrm{dia}^{-}\right.$ $\left.{ }^{1}\right)$;

$\mathrm{G}=$ densidade do fluxo de calor do solo (MJ $\left.\mathrm{m}^{-2} \mathrm{dia}^{-1}\right)$;

$t_{\text {méd }}=$ temperatura do ar média diária a $2 \mathrm{~m}$ de altura $\left({ }^{\circ} \mathrm{C}\right)$;

$\mathrm{u}_{2}=$ velocidade do vento a $2 \mathrm{~m}$ de altura $\left(\mathrm{m} \mathrm{s}^{-}\right.$ $\left.{ }^{1}\right)$;

$\mathrm{e}_{\mathrm{s}}=$ pressão do vapor de saturação $(\mathrm{kPa})$;

$\mathrm{e}_{\mathrm{a}}=$ pressão do vapor atual $(\mathrm{kPa})$;

$\Delta=$ declividade da curva de pressão do vapor $\left(\mathrm{kPa}{ }^{\circ} \mathrm{C}^{-1}\right)$;

$\gamma=$ constante psicrométrica $\left(\mathrm{kPa}^{\circ} \mathrm{C}^{-1}\right)$.

Onde as variáveis meteorológicas, temperatura $\left(\mathrm{T},{ }^{\circ} \mathrm{C}\right)$, umidade relativa do ar (UR, \%), radiação solar global (Rg, $\mathrm{MJ} \mathrm{m}^{-2} \mathrm{dia}^{-}$ ${ }^{1}$ ) e de velocidade do vento a dois metros de altura $\left(\mathrm{u}_{2}, \mathrm{~m} \mathrm{~s}^{-1}\right)$ foram obtidas por meio de uma estação meteorológica automática da Agência Pernambucana de Água e Clima (www.apac.pe.gov.br), situada na área da Fazenda Saco, município de Serra Talhada-PE, para o período do estudo.

\section{RESULTADOS E DISCUSSÃO}

Observa-se que o comportamento dos valores médios de ETo determinados pelo método da evapotranspiração de referência de Penman-Monteith (ETopM), em relação aos resultados obtidos pelo método de Tanque Classe " $A$ ", utilizando os diferentes valores de 
Kp (Tabela 1) pode-se constatar que todos os métodos superestimaram a ETopm, tendo destaque o valor de ETo(TCA) Kpsnyder, que apresentou a maior diferença em relação ao de ETopm, chegando a ser de 76\%, o quê equivale a aproximadamente $58 \mathrm{~mm}$ (Figura 2).

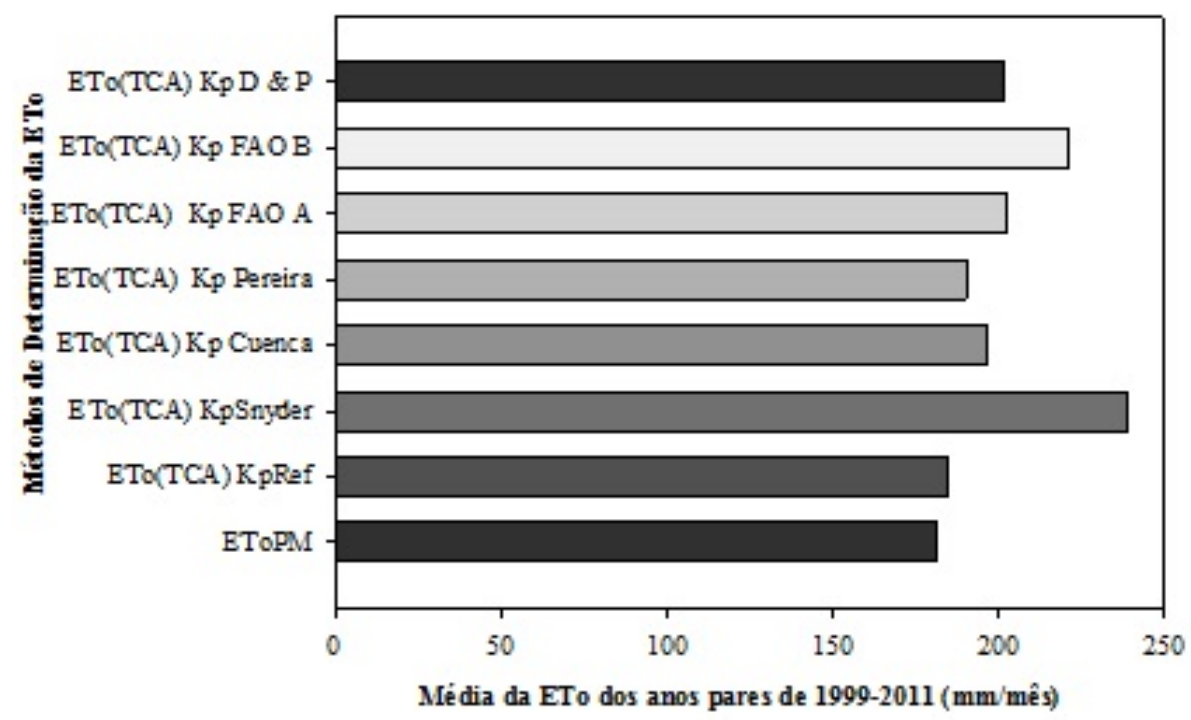

Figura 2. Média mensal da evapotranspiração de referência estimadas pelos métodos de Penman-Monteith FAO 56 e do Tanque do Classe "A" considerando diferentes os valores de Kp, para a cidade de Serra Talhada, Pernambuco.

Também, merece destaque o comportamento dos resultados obtidos pelos (métodos da evapotranspiração de referência pelo método do Tanque Classe “A” (ETo(TCA)) e coeficiente do tanque pela FAO caso B (Крғаов), ETo(ТСа) e coeficiente do tanque pela FAO caso A (KpFAOA) e ETo(TCA) método do tanque Classe “A” de Doorenbos e Pruitt (1977) (KpD\&P), com 48, 24 e 23\% a mais da ETC 20,5 mm, respectivamente (Figura 2).

Para os demais métodos, a ETo(TCA) Kpref foi a que mais se aproximou dos valores de ETopm, possuindo somente $3 \%$ a mais ( $3 \mathrm{~mm}$ ), seguido pelos valores de ETo(TCA) com os Kppereira e Kpsnyder, que tiveram 9 e $17 \%$, o que gera uma diferença de aproximadamente 9 e 15 $\mathrm{mm}$, respectivamente (Figura 2).

Cunha et al. (2011), em trabalho executado em Botucatu, São Paulo, verificou que em campo houve somente comportamento de superestimativa dos valores da ETo(TCA), determinada pelos diferentes valores dos métodos de Kp em relação ao ETopm. Para o método proposto por Doorenbos e Pruitt (1977) que alcançou 12,4\% no período seco da região e $26,7 \%$ no chuvoso. Os demais métodos subestimaram a ETo(TCA) em relação a ETopm, apresentando valores de 18,6 e 10,8\% (CUENCA, 1989); 15,4 e 8,6\% (SNYDER, 1992); 25,8 e 27,0\% (PEREIRA et al., 1995); e 13,7 e 6,0\% (ALLEN et al., 1998), durante o período seco e chuvoso, respectivamente.

Quanto aos valores de ETc obtidos pelos diferentes métodos, com a utilização do Kc para cada fase fenológica do girassol, pode-se constatar um valor médio da ЕTсрм de $405 \mathrm{~mm}$ nas condições de Serra Talhada-PE (Tabela 2), assemelhando-se ao obtido por Cavalcante Junior et al. (2013), de 442 mm, na cidade de Apodi, no Rio Grande do Norte, que é também uma a região classificada por um clima muito quente e semiárido, conforme Köppen.

Tabela 2. Desempenho dos valores de Kp de sete métodos: Kpref, Pereira et al. (2014); Kpsnyder, Snyder (1992); Kpcuenca, Cuenca (1989); Kppereira, Pereira et al. (1995); KpғaOA e Kpғaob, Allen et al. (1998); e KpD\&p, Doorenbos e Pruitt (1977), na determinação dos valores de ETc da cultura do 
girassol (Helianthus annuus L.), ao longo dos possíveis ciclos durante o ano, em Serra Talhada, Pernambuco.

\begin{tabular}{|c|c|c|c|c|}
\hline Métodos & $\begin{array}{c}\text { Ciclo I } \\
\text { (Jan-Mar) }\end{array}$ & $\begin{array}{c}\text { Ciclo II } \\
\text { (Abr-Jun) }\end{array}$ & $\begin{array}{c}\text { Ciclo III } \\
\text { (Jun-Set) }\end{array}$ & $\begin{array}{l}\text { Ciclo IV } \\
\text { (Set-Dez) }\end{array}$ \\
\hline ETСрм & $394,89^{\mathrm{Bb}}$ & $285,99^{\mathrm{Ba}}$ & $369,94^{\mathrm{Ba}}$ & $568,88^{\mathrm{Ab}}$ \\
\hline $\mathrm{ETc}_{(\mathrm{TCA})} \mathrm{K} p_{\text {Ref }}$ & $448,42 \mathrm{ABab}$ & $293,33^{\text {Ca }}$ & $380,24^{\text {BCa }}$ & $524,43^{\mathrm{Ab}}$ \\
\hline $\mathrm{ETC}_{\text {(TCA) }} \mathrm{Kp}_{\text {Snyder }}$ & $514,36^{\mathrm{Ba}}$ & $359,31^{\mathrm{Ca}}$ & $507,23^{\mathrm{Ba}}$ & $752,88^{\mathrm{Aa}}$ \\
\hline $\mathrm{ETC}_{(\mathrm{TCA})} \mathrm{Kp}_{\text {Cuenca }}$ & 456,91 ABab & $312,61^{\mathrm{Ca}}$ & $421,98^{\text {BCab }}$ & $569,66^{\mathrm{Ab}}$ \\
\hline $\mathrm{ETC}_{(\mathrm{TCA})} \mathrm{Kp}_{\text {Pereira }}$ & 433,39 Bab & $298,93^{\mathrm{Ba}}$ & $391,98^{\mathrm{Bb}}$ & $575,47^{\mathrm{Ab}}$ \\
\hline $\mathrm{ETC}_{(\mathrm{TCA})} \mathrm{Kp}_{\mathrm{FAO}}$ & 460,56 ABab & 332,90 Ва & $431,23^{\mathrm{Bab}}$ & $589,93^{\mathrm{Ab}}$ \\
\hline $\mathrm{ETc}_{(\mathrm{TCA})} \mathrm{Kp}_{\mathrm{FAO}}$ & $506,25^{\mathrm{ABab}}$ & 368,59 Ва & $470,16^{\mathrm{Bab}}$ & $634,21^{\mathrm{Ab}}$ \\
\hline $\mathrm{ETC}_{(\mathrm{TCA})} \mathrm{Kp}_{\mathrm{D} \& \mathrm{P}}$ & $451,75^{\mathrm{Bab}}$ & $319,41^{\text {Ва }}$ & $429,10^{\mathrm{Bab}}$ & $606,43^{\mathrm{Ab}}$ \\
\hline
\end{tabular}

*Médias seguidas pelas mesmas letras maiúsculas na horizontal e minúsculas na vertical não diferem estatisticamente entre si, ao nível de $5 \%$ de probabilidade pelo teste de Tukey.

A partir das simulações dos valores de ETc para cada ciclo, observa-se que o método da ETсрм diferenciou-se somente dos valores de ETc(TCA) Kpsnyder nos ciclos I e IV. E que os valores médios de ETc(TCA) Крғаов e ETc(TCA) Kpsnyder destacaram-se, apresentando as maiores médias entre os métodos e em todas as simulações de ciclo, desta forma essas metodologias podem causar uma superestimava da lâmina de irrigação para cultura do girassol, mesmo não tendo apresentado diferença estatística em relação ao método padrão (Tabela 2).

Pereira et al. (2014) constataram efeitos semelhantes, tendo eles não aconselhado o uso dos Kp dos métodos de Snyder (1992) e FAO caso B (ALLEN et al., 1998) para essa a cidade de Serra Talhada-PE, devido a superestimação da necessidade hídrica das culturas avaliadas. Braga et al. (2008), no Submédio do Vale do São Francisco, também mostraram que o método de Snyder não é adequado para a região, por também superestimar a lâmina de irrigação.

Esse efeito, pode estar associado ao fato que esses métodos foram desenvolvidos para regiões com condições climáticas diferentes da região do presente estudo, tornando necessário calibrações para cada local, de acordo com as recomendações da FAO (ALLEN et al., 1998).

Por sua vez, os métodos de $\mathrm{ETc}_{(\mathrm{TCA}) \text {, }}$ Kpref e ETc(TCA), Kppereira foram os que apresentaram valores mais próximos aos da ETсрм, podendo serem utilizados para estimar a evapotranspiração do girassol nessa localidade e em locais com características climáticas semelhantes dessa região, especialmente quando não é possível obter todas as variáveis meteorológicas locais.

O bom comportamento da ETc (TCA) KpRef está no fato de ter sido obtido para essa região, por meio de uma calibração que utilizou de dados diários de ETopm e ETCA de Serra Talhada, o que possibilitou Kp de maior confiabilidade na determinação da ETc (PEREIRA et al., 2014). E no caso do método de Pereira et al. (1995), o seu bom desempenho foi, da mesma forma, constatado na cidade de Juazeiro-BA, que também está localizada no Semiárido brasileiro (BRAGA et al., 2008), e para cidades de localidades que se diferenciam climaticamente ao do presente estudo, como é o caso das cidades de Piracicaba-SP (SENTELHAS; FOLEGATTI, 2003) e Campos dos Goytacazes-RJ (ESTEVES et al., 2009).

Em relação as simulações dos ciclos do girassol ao longo do ano, as maiores médias foram observadas no ciclo IV (Tabela 2), que compreendeu os meses de setembro a dezembro, período que apresentou comportamento médio das variáveis meteorológicas, dos anos de 1999 a 2011, de baixos índices pluviométricos e de umidade relativa, $70 \mathrm{~mm}$ e $52 \%$, com altas faixas de temperatura média e radiação, $27^{\circ} \mathrm{C}$ e $30 \mathrm{MJ}$ $\mathrm{m}^{-2} \mathrm{dia}^{-1}$, respectivamente, proporcionando alta demanda atmosférica, elevando assim a ETc do girassol.

Para todos os métodos de obtenção da ETc, apenas o ciclo IV se diferenciou estatisticamente dos ciclos II e III (Tabela 2). 
Esses últimos ciclos estão inseridos em faixas mais amenas de temperatura média, 24,3 e 23,9 ${ }^{\circ} \mathrm{C}$, níveis médios de precipitação pluviométrica, 215 e $67 \mathrm{~mm}$, baixas taxas de radiação, 23 e $22 \mathrm{MJ} \mathrm{m}^{-2}$ dia $^{-1}$, e elevadas faixas de umidade relativa, 73 e $67 \%$, respectivamente, que provoca valores mais baixos de evapotranspiração, quando comparada ao ciclo IV.

E ao comparar os ciclos I e IV, observouse que houve diferença estatística entre os métodos ETcpm, $\mathrm{ETc}_{(\mathrm{TCA})} \mathrm{Kpsnyder,} \mathrm{ETc}_{(\mathrm{TCA})}$ KpPereira e ETc(TCA) KpD\&P, que está associada as distintas condições climáticas de cada ciclo, como já citado, o período de tempo que compreende o ciclo IV possui características que proporciona altas taxa de evapotranspiração, sendo superior aos valores das variáveis meteorológicas do período do ciclo I, contido nos meses de janeiro a março, que possui valores médios mais amenos de temperatura média e radiação, $25,8^{\circ} \mathrm{C}$ e $28 \mathrm{MJ}$ $\mathrm{m}^{-2}$ dia $^{-1}$, e altas faixas de precipitação pluviométrica e umidade relativa $403 \mathrm{~mm}$ e $70 \%$, respectivamente.

Os índices estatísticos utilizados para avaliar o desempenho dos valores de ETc do girassol, estimados pelos dados médios mensais de Kp podem serem observados na Tabela 3, onde constata-se que houve uma correlação positiva entre os valores de ETc(TCA) e ETCPм, variando de 0,87 a 0,90 , demonstrando que os valores dos ETC(TCA) proporcionaram elevadas estimativas de precisão na ETc do girassol.

Tabela 3. Coeficiente de correlação (r), índice de concordância (d), erro médio de estimativa (MBE), raiz quadrada do quadrado médio do erro (RQME) e índice de desempenho (c) dos valores de ETc(TCA) obtido por valores de sete métodos de estimativa do Kp: Kpref, Pereira et al. (2014); Kpsnyder, Snyder (1992); Kpcuenca, Cuenca (1989); Kppereira, Pereira et al. (1995); Крғаон e Крғаов, Allen et al. (1998); e KpD\&p, Doorenbos e Pruitt (1977), em Serra Talhada, Pernambuco.

\begin{tabular}{|c|c|c|c|c|c|c|c|}
\hline Índices & $\begin{array}{c}\text { ETc }_{(\text {TCA) }} \\
\text { Kp }_{\text {Ref }}\end{array}$ & $\begin{array}{l}\text { ETc }_{\text {(TCA) }} \\
\text { Kp }_{\text {Snyder }}\end{array}$ & 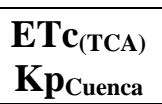 & $\begin{array}{l}\mathbf{E T c}_{(\mathrm{TCA})} \\
\text { Kp } \text { pereira }\end{array}$ & $\begin{array}{l}\mathbf{E T c}_{(\mathrm{TCA})} \\
\text { Кр } \text { paO A }\end{array}$ & $\begin{array}{l}\mathbf{E T c}_{(\mathrm{TCA})} \\
\text { Кр } \text { pAO B }\end{array}$ & $\begin{array}{c}\text { ETc }_{(\text {TCA) }} \\
\text { Kp }_{\text {D\&P }}\end{array}$ \\
\hline$r$ & 0,87 & 0,90 & 0,89 & 0,90 & 0,90 & 0,89 & 0,90 \\
\hline $\mathrm{d}$ & 0,91 & 0,79 & 0,91 & 0,94 & 0,90 & 0,83 & 0,91 \\
\hline MBE & 6,68 & 128,52 & 35,37 & 20,02 & 48,73 & 89,88 & 46,75 \\
\hline RQME & 67,02 & 145,23 & 70,49 & 60,43 & 76,32 & 107,43 & 73,45 \\
\hline C & 0,79 & 0,71 & 0,81 & 0,84 & 0,81 & 0,74 & 0,82 \\
\hline Classificação & $\begin{array}{c}\text { Muito } \\
\text { Bom }\end{array}$ & Bom & $\begin{array}{c}\text { Muito } \\
\text { Bom }\end{array}$ & $\begin{array}{c}\text { Muito } \\
\text { Bom }\end{array}$ & $\begin{array}{c}\text { Muito } \\
\text { Bom }\end{array}$ & Bom & $\begin{array}{l}\text { Muito } \\
\text { Bom }\end{array}$ \\
\hline
\end{tabular}

Esses resultados foram superiores aos obtidos por Pereira et al. (2014), que variaram de 0,16 a 0,76 para diferentes culturas, também, avaliando os valores de ETc e inferiores ao desempenho encontrado por Braga et al. (2008), que ficaram entre 0,91 a 0,99 , quando eles avaliaram a determinação da ETo.

Os valores dos Kp de Snyder (SNYDER, 1992) e FAO caso B (ALLEN et al., 1998), proporcionaram os menores resultados do índice exatidão (índice de concordância, d), apresentando 0,79 e 0,83 , respectivamente, o qual indica o grau de exatidão entre os valores de $\operatorname{ETc}_{\text {(TCA) }}$ e ETсрм. O d varia de 0 a 1, sendo que quanto mais próximo de 1 maior é o grau de exatidão (WILLMOTT, 1985) da estimativa

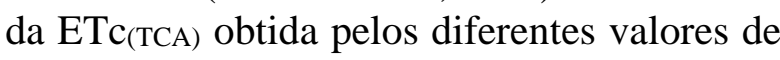
Kp. Por outro lado, o Kp proposto por Pereira et al. (1995) foi o que apresentou maior grau de exatidão (0,94), seguido pelo Kpref (PEREIRA et al., 2014), Kpcuenca (CUENCA, 1989) e de Doorenbos e Pruitt (1977) (KpD\&P), que tiveram o mesmo valor $(0,91)$.

Nas mesmas condições climáticas do presente estudo, Pereira et al. (2014), obteve valores de $\operatorname{ETc}_{\text {(TCA) }}$ entre 0,43 a 0,85 , que é muito abaixo ao observado neste trabalho.

O erro médio de estimativa (MBE), utilizados para quantificar as sub ou superestimativas dos valores de $\mathrm{ETC}_{(\mathrm{TCA}) \text {, }}$ estimada pelos valores de $\mathrm{Kp}$ a longo prazo, 


\section{SEMIÁRIDO BRASILEIRO}

oscilaram entre 6,68 $\mathrm{mm} \mathrm{ciclo}^{-1}$ (ETc(TCA) Kpref) a 128,52 mm ciclo $^{-1}$ (ETc(TCA) Kpsnyder), melhor e pior desempenho, concomitantemente, ilustrando um ajuste anual na lâmina de irrigação a ser aplicada na cultura, de acordo com o método a ser escolhido. Enquanto que a raiz quadrada do quadrado do erro médio (RQME), que fornece subsídios sobre o desempenho destes valores a curto prazo, oscilou entre $60,43 \mathrm{~mm}$ ciclo $^{-1}$ $\left(\mathrm{ETc}(\mathrm{TCA})\right.$ Kppereira) a $145,23 \mathrm{~mm}_{\text {ciclo }^{-1}}$ (ETc(TCA) Kpsnyder), indicando uma variabilidade do desempenho na estimativa da ETc durante o ano, para cultura do girassol.

Pereira et al. (2014), obtiveram um comportamento semelhante, onde também evidenciaram que os piores desempenhos ficaram atrelados a ETc(TCA) Kpsnyder, tanto para o MBE como pra o RQME, superestimando de forma significativa as lâminas de irrigação. E como melhores desempenhos, ficaram para a ETc(TCA) KpRef e ETc(TCA) Kppereira para o MBE e ETc(TCA) KpRef e ETc(TCA) KpғaOa no RQME. A utilização do método de determinação do Kp de Pereira et al. (1995), proporcionou para Braga et al. (2008) os melhores resultados de MBE e RQME, em Juazeiro-BA, o que reforça a eficiência desta metodologia para esses dois ambientes, que estão inseridos no Semiárido brasileiro.

Por fim, a classificação do desempenho dos valores de Kp na estimativa da ETC(TCA) do girassol, pelo índice de confiança (c), ficou entre "bom", para ETc(TCA) Kpsnyder e ETc(TCA) Крғаов, e "muito bom", nos demais métodos. Estes resultados associaram-se diretamente ao desempenho dos valores estimados de $\mathrm{Kp}$, procedendo em um baixo desempenho nas

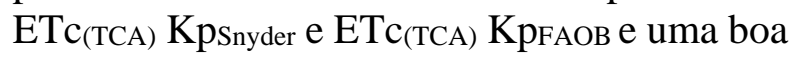
estimativa nas $\operatorname{ETc}_{(\mathrm{TCA})} \mathrm{Kpref}_{\text {e }} \operatorname{ETc}_{(\mathrm{TCA})}$ KpPereira.

\section{CONCLUSÕES}

Na ausência de variáveis meteorológicas para determinar a evapotranspiração da cultura (ETc), a partir da evapotranspiração de referência (ETo - Penman-Monteith FAO 56), indica-se o método do Tanque Classe " $\mathrm{A}$ ” para o Vale do Pajeú, onde os Kpref e Kppereira auxiliaram na melhor estimativa da ETc do girassol. Já a utilização dos valores de Kp obtidos pelos métodos de Snyder e FAO caso B não são recomendados para essa localidade, podendo ser aplicáveis em outras regiões, desde que para isso, haja preliminarmente uma calibração e validação local, semelhante ao que foi realizado nesse trabalho.

\section{REFERÊNCIAS BIBLIOGRÁFICAS}

ALCARAS, L. M. A.; ROUSSEAUX, M. C.; SEARLES, P. S. Responses of several soil and plant indicators to post-harvest regulated deficit irrigation in olive trees and their potential for irrigation scheduling. Agricultural

Water Management, Amsterdam, v. 171, p. 10-20, 2016.

ALLEN, R. G.; PEREIRA, L. S.; RAES, D. Crop evapotranspiration. Guidelines for computing crop water requirements. Rome: Irrigation and Drainage Paper 56, FAO, 1998. $300 \mathrm{p}$.

ALVARES, C. A.; STAPE, J. L.; SENTELHAS, P. C.; MORAES, G. J. L.; SPAROVEK, G. Köppen's climate classification map for Brazil. Meteorologische Zeitschrift, Stuttgart, v. 22, n. 6, p. 711-728, 2013.

BRAGA, M. B.; CALGARO, M.; MOURA, M. S. B.; SILVA, T. G. F. Coeficientes do tanque classe "A" para estimativa da evapotranspiração de referência na região do Vale do Submédio São Francisco, estado da Bahia. Revista Brasileira de Agrometeorologia, Santa Maria, v. 16, n. 1, p. 49-57, 2008.

CAMARGO, A. P.; SENTELHAS, P. C. Avaliação do desempenho de diferentes métodos de estimativa da evapotranspiração potencial no Estado de São Paulo, Brasil. Revista Brasileira de Agrometeorologia, Santa Maria, v. 5, n. 1, p. 89-97, 1997. 
CAVALCANTE JUNIOR, E. G.; MEDEIROS, J. F.; MELO, T. K.; ESPINOLA SOBRINHO, J.; BRISTOT, G.; ALMEIDA, B. M. Necessidade hídrica da cultura do girassol irrigado na chapada do Apodi. Revista Brasileira de Engenharia Agrícola e Ambiental, Campina Grande, v. 17, n. 3, p. 261-267, 2013.

CUENCA, R. H. Irrigation system design: an engineering approach. New Jersey: PrenticeHall, 1989, 133 p.

CUNHA, A. R. Coeficiente do tanque Classe A obtido por diferentes métodos em ambiente protegido e no campo. Ciências Agrárias, Londrina, v. 32, n. 2, p. 451-464, 2011.

DOORENBOS, J.; PRUITT, W. O. Crop Water Requirements. Rome: FAO, 1977. 168 p. (Irrigation and Drainage Paper, 24).

ESTEVES, B. S.; MENDONÇA, J. C.; SOUSA, E. F.; BERNARDO, S. Avaliação do Kt para estimativa da evapotranspiração de referência (ETo) em Campos dos Goytacazes, RJ. Revista Brasileira de Engenharia Agrícola e Ambiental, Campina Grande, v. 14, n. 3, p. 274-278, 2009.

FREITAS, C. A. S.; SILVA, A. R. A.; BEZERRA, F. M. L.; ANDRADE, R. R.; MOTA, F. S. B.; AQUINO, B. F. Crescimento da cultura do girassol irrigado com diferentes tipos de água e adubação nitrogenada. Revista Brasileira de Engenharia Agrícola e Ambiental, Campina Grande, v. 16, n. 10, p. 1031-1039, 2012.

OLIVEIRA, R. A.; TAGLIAFERRE, C.; SEDIYAMA, G. C.; MATERAM, F. J. V.; CECON, P. R. Desempenho do Irrigâmetro na estimativa da evapotranspiração de referência.

Revista Brasileira de Engenharia Agrícola e Ambiental, Campina Grande, v. 12, n. 2, p.166-173, 2008.

PEREIRA, A. R.; SEDIYAMA, G. C.; NOVA, N. A. V. Evapotranspiração. Campinas: Fundag, 2013. 323 p.
PEREIRA, A. R.; VILLA NOVA, N.; PEREIRA, A. S.; BARBIERI, V. A model for class-A pan coefficient. Agricultural and Forest Meteorology, Amsterdam, v. 76, p. 7582, 1995.

PEREIRA, P. C.; SILVA, T. G. F.; SILVA, S. M. S.; CRUZ NETO, J. F.; MORAIS, J. E. F. Avaliação e aplicabilidade do coeficiente do tanque classe a no Médio Pajeú, Pernambuco. Revista Caatinga, Mossoró, v. 27, n. 1, p. 131-140, 2014.

SENTELHAS, P. C.; FOLEGATTI, M. V. Class A pan coefficients (Kp) to estimate daily reference evapotranspiration (ETo). Revista Brasileira de Engenharia Agrícola e Ambiental, Campina Grande, v. 7, n. 1, p. 111-115, 2003.

SILVA, M. L. O.; FARIA, M. A.; MORAIS, A. R.; ANDRADE, G. P.; LIMA, E. M. C. Crescimento e produtividade do girassol cultivado na entressafra com diferentes lâminas de água. Revista Brasileira de Engenharia Agrícola e Ambiental, Campina Grande, v. 11, n. 5, p. 482- 488, 2007.

SNYDER, R. L. Equation for evaporation pan to evapotranspiration conversions. Journal of the Irrigation and Drainage Engineering, New York, v. 118, p. 977-980, 1992.

SOUSA, I. F. SILVA, V. P. R.; SABINO, F. G.; SILVA, B. K. N.; AZEVEDO, P. V. Evapotranspiração de referência nos perímetros irrigados do Estado de Sergipe. Revista Brasileira de Engenharia Agrícola e Ambiental, Campina Grande, v. 14, n. 6, p. 633-644, 2010.

WILLMOTT, C. J. Statistics for the evaluation and comparison of models. Journal of Geophysical Research Oceans, $\quad$ n. $\quad 90$, p. 89959005, 1985 